\title{
ARTICLE
}

Received 15 Jul 2014 | Accepted 30 Sep 2014 | Published 14 Nov $2014 \quad$ DOl: 10.1038/ncomms6424

\section{Explaining why simple liquids are quasi-universal}

\author{
Andreas K. Bacher ${ }^{1}$, Thomas B. Schrøder ${ }^{1} \&$ Jeppe C. Dyre ${ }^{1}$
}

It has been known for a long time that many simple liquids have surprisingly similar structure as quantified, for example, by the radial distribution function. A much more recent realization is that the dynamics are also very similar for a number of systems with quite different pair potentials. Systems with such non-trivial similarities are generally referred to as 'quasi-universal'. From the fact that the exponentially repulsive pair potential has strong virial potential-energy correlations in the low-temperature part of its thermodynamic phase diagram, we here show that a liquid is quasi-universal if its pair potential can be written approximately as a sum of exponential terms with numerically large prefactors. Based on evidence from the literature we moreover conjecture the converse, that is, that quasi-universality only applies for systems with this property.

\footnotetext{
${ }^{1}$ DNRF Center 'Glass and Time', IMFUFA, Department of Sciences, Roskilde University, Postbox 260, DK-4000 Roskilde, Denmark. Correspondence and requests for materials should be addressed to J.C.D. (email: dyre@ruc.dk).
} 
A cornerstone of liquid-state theory is the fact that the hardsphere (HS) model gives an excellent representation of simple liquids. This was discussed in 1959 by Bernal ${ }^{1}$, and the first liquid-state computer simulations at the same time by Alder and Wainwright ${ }^{2}$ likewise studied the HS system. Following this the HS model has been the fundament of liquid-state theory, in particular since the 1970s when perturbation theory matured into its present form ${ }^{3-5}$. The HS model is usually invoked to explain the fact that many simple liquids have very similar structure. This cannot explain, however, the more recent observation of dynamic quasi-universality ${ }^{6-14}$ or why some mathematically simple pair potentials violate quasiuniversality ${ }^{15-19}$.

An early hint of dynamic quasi-universality was provided by Rosenfeld $^{6}$ who showed that the diffusion constant is an almost universal function of the excess entropy (the entropy minus that of an ideal gas at the same temperature and density). This finding did not attract a great deal of attention at the time, but the last decade has seen renewed interest in excess-entropy scaling ${ }^{7,8}$ and, more generally, in the striking similarities of the structure and dynamics of many simple model liquids ${ }^{9-14}$. Thus Heyes and Branka $^{9,11,20-23}$ and others have documented that inverse powerlaw (IPL) systems with different exponents have similar structure and dynamics, Medina-Noyola and co-workers ${ }^{10,13,14}$ have established that quasi-universality extends to the dynamics for Newtonian as well as Brownian equations of motion, Scopigno and co-workers ${ }^{21}$ have documented HS-like dynamics in liquid gallium studied by quasi-elastic neutron scattering, and Liu and co-workers ${ }^{12}$ have suggested a mapping of a soft-sphere system's dynamics to that of the HS system.

Further examples of similarities between apparently quite different systems, identified throughout the years but still largely unexplained, include: the Young-Andersen ${ }^{24}$ approximate scaling principle according to which two systems that have the same radial distribution function-even at different thermodynamic state points-also have the same dynamics; the fact that different systems have similar order-parameter maps in the sense of Debenedetti and co-workers ${ }^{8,25}$, that is, when the relevant orientational order parameter is plotted against the translational one, almost identical curves result; the Lindemann and other melting rules ${ }^{26,27}$; freezing rules like Andrade's finding ${ }^{28}$ that freezing initiates when the reduced viscosity upon cooling reaches a certain value or the Hansen-Verlet ${ }^{29,30}$ rule that a liquid freezes when the maximum structure factor reaches 2.85 .

In the literature the term 'quasi-universality' refers to the general observation that different simple systems have very similar physics as regards structure and dynamics, but before proceeding we need to define the term precisely. To do this, recall first the definition of so-called reduced quantities. These are quantities made dimensionless by dividing by the appropriate combination of the following three units: the length unit is $\rho^{-1 / 3}$ where $\rho$ is the number density, the energy unit is $k_{\mathrm{B}} T$ where $T$ is the temperature, and the time unit is $\rho^{-1 / 3} \sqrt{m / k_{B} T}$ for Newtonian dynamics where $m$ is the particle mass (for Brownian dynamics a different time unit is used ${ }^{31,32}$ ). Henceforth, reduced quantities are denoted by a tilde, for instance the reduced pair distance is defined by $\tilde{r} \equiv \rho^{1 / 3} r$.

By quasi-universality we shall mean the property of many simple model liquids that knowledge of a single quantity characterizing the structure or dynamics in reduced units is enough to determine all other reduced-unit structural and dynamic quantities to a good approximation. Note that a special case of this is Rosenfeld's observation that the excess entropy over $k_{\mathrm{B}}$, a structural quantity, determines the reduced diffusion constant. Likewise, the Young-Andersen ${ }^{33}$ approximate scaling principle is a consequence of quasi-universality as defined here- as these authors expressed it '... certain dynamical properties are very insensitive to large changes in the interatomic potential that leave the pair correlation function largely unchanged'.

It is straightforward to show that if quasi-universality applies, the other points mentioned above also follow. The fundamental questions are: which systems are quasi-universal? what causes quasi-universality? The conventional explanation of quasiuniversality starts from the fact that the HS system provides an excellent reference system for structure calculations $6,10,12-14,34$. Approximate theories of liquid dynamic properties like renormalized kinetic theory or mode-coupling theory in its simplest version predict that the dynamics is uniquely determined by the static structure factor, that is, that structure determines dynamics. This reasoning led to the search for and discovery of dynamic quasi-universality back in 2003 (refs 10,24).

The dynamics of the HS system consists of constant-velocity free-particle motion interrupted by infinitely fast collisions, which is quite different from the continuous motion described by Newton's laws for smooth potentials. Thus it is far from physically obvious why the HS explanation of quasi-universal structure extends to the dynamics. Moreover, while the HS explanation does account for the finding that, for example, the Gaussian-core model $^{15,35,36}$ violates quasi-universality-a model without harsh repulsions except at low temperatures-it cannot explain why some strongly repulsive or hard-core pair-potential systems violate quasi-universality. Examples of such systems are the LennardJones (LJ) Gaussian model ${ }^{17}$ and the Jagla model ${ }^{37}$. Finally, the one-component plasma (OCP) model is well-known to be quasiuniversal $^{6}$, but it is not intuitively clear when and why the gently varying Coulomb force can be well-approximated by the harsh HS interaction.

In this paper we do not use the HS reference system. In a continuation of recent works ${ }^{38-40}$ we take a different approach to quasi-universality by showing that any pair potential, which can be approximated by a sum of exponential terms with numerically large prefactors, is quasi-universal. This introduces the 'EXP quasi-universality class' of pair potentials. Based on the available evidence from the literature we moreover conjecture the converse, that is, that all quasi-universal systems are in the EXP quasiuniversality class.

\section{Results}

The exponentially repulsive pair potential. We study below the monatomic system described by the purely repulsive 'EXP' pair potential

$$
v_{\operatorname{EXP}}(r, \varepsilon, \sigma)=\varepsilon e^{-r / \sigma} .
$$

This was discussed already by Born and Meyer ${ }^{41}$ and by Buckingham $^{42}$ in the context of a pair potential with an exponentially repulsive term plus an $r^{-6}$ attractive term; note also that the well-known Morse pair potential is a difference of two exponentials ${ }^{43}$. The EXP pair potential may be justified physically as reflecting the overlap of electron wavefunctions in conjunction with the fact that bound-state wavefunctions decay exponentially in space ${ }^{41}$. Although the EXP pair potential since 1932 has been used occasionally in computer simulations and for interpretation of experiments $^{40,44-48}$, it never became a standard pair potential like the LJ and Yukawa pair potentials ${ }^{29,49}$. Given the mathematical simplicity of the EXP function this may seem surprising, but a likely explanation is that no system in nature is believed to be welldescribed by this purely repulsive pair potential. The present paper suggests that it may nevertheless be a good idea to regard the EXP pair potential as the fundamental building block of the physics of simple liquids, much like the exponential function in the Fourier and Laplace transform theories of pure mathematics. 


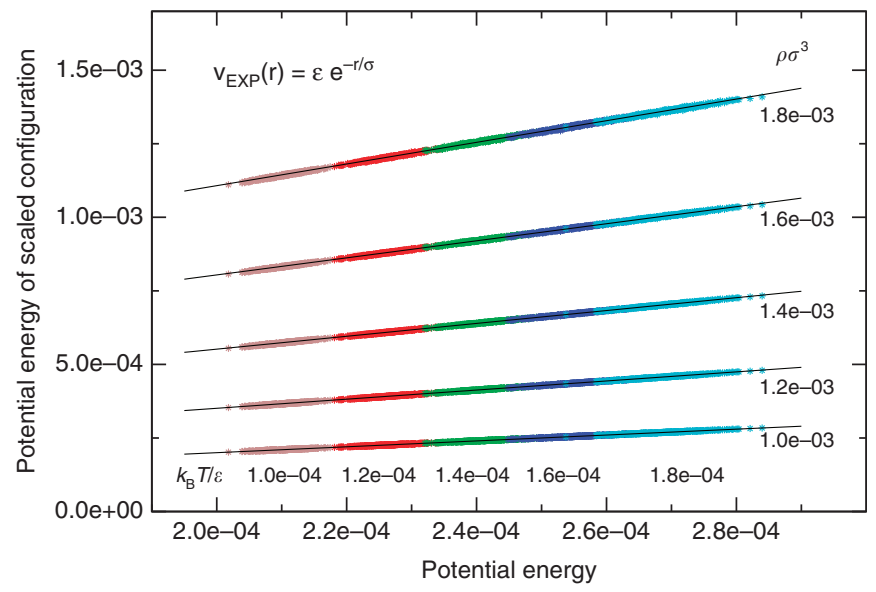

Figure 1 | Scatter plots of potential energies per particle of the EXP

system. At the state point with density $\rho \sigma^{3}=1.0 \times 10^{-3}$ and temperature $k_{\mathrm{B}} T / \varepsilon=1.0 \times 10^{-4}$ several configurations were selected from an equilibrium simulation and scaled uniformly to four higher densities (the light brown points); the same was done for four other temperatures at this density. The figure shows that there are very strong correlations between scaled and unscaled potential energies, with a scaling factor that only depends on the two densities involved. The lines are best fits to the green data points, that is, those generated from simulations at temperature $k_{\mathrm{B}} T / \varepsilon=1.4 \times 10^{-4}$. This figure validates the hidden-scale-invariance property of the EXP pair-potential system as expressed below in equation (4).

Our simulations focused on the low-temperature part of the thermodynamic phase diagram, that is, where

$$
k_{B} T \ll \varepsilon .
$$

The potential energy as a function of all the particle coordinates $\mathbf{R} \equiv\left(\mathbf{r}_{1}, \ldots, \mathbf{r}_{N}\right)$ is denoted by $U_{\mathrm{EXP}}(\mathbf{R})$. Figure 1 shows scatter plots of $U_{\text {EXP }}(\mathbf{R})$ for several configurations $\mathbf{R}$ plotted versus the potential energies of the same configurations scaled uniformly to a different density, that is, $U_{\mathrm{EXP}}(\mathbf{R})$ plotted versus $U_{\mathrm{EXP}}(\lambda \mathbf{R})$ in which $\lambda^{3}$ is the ratio of the two densities in question. The figure was constructed by simulating five temperatures at the density $\rho \sigma^{3}=1.0 \times 10^{-3}$ (lower line, different colours); at each of these five state points, configurations were selected and scaled to four higher densities. We see that there are very strong correlations between the potential energies of scaled and unscaled configurations.

As shown elsewhere strong correlations between scaled and unscaled potential energies are characteristic for systems that have strong correlations between their virial and potential energy thermal equilibrium fluctuations at the relevant state points ${ }^{31}$. Systems with this property include not only 'atomic' pairpotential systems like the IPL, Yukawa, and LJ systems and so on, but also a number of rigid molecular model systems and even the flexible LJ chain model ${ }^{50,51}$. Such systems were previously referred to as 'strongly correlating', but are now called Roskildesimple or just Roskilde systems ${ }^{52-57}$, which avoids confusion with strongly correlated quantum systems.

The strength of the virial potential-energy correlations of the EXP system is reported in Fig. 2a for a large number of state points. If virial and potential energy are denoted by $W$ and $U$, respectively, and sharp brackets denote canonical constantvolume (NVT) averages, the colour coding of the figure gives the Pearson correlation coefficient $R$ defined ${ }^{58}$ by

$$
R=\frac{\langle\Delta W \Delta U\rangle}{\sqrt{\left\langle(\Delta U)^{2}\right\rangle\left\langle(\Delta W)^{2}\right\rangle}} .
$$
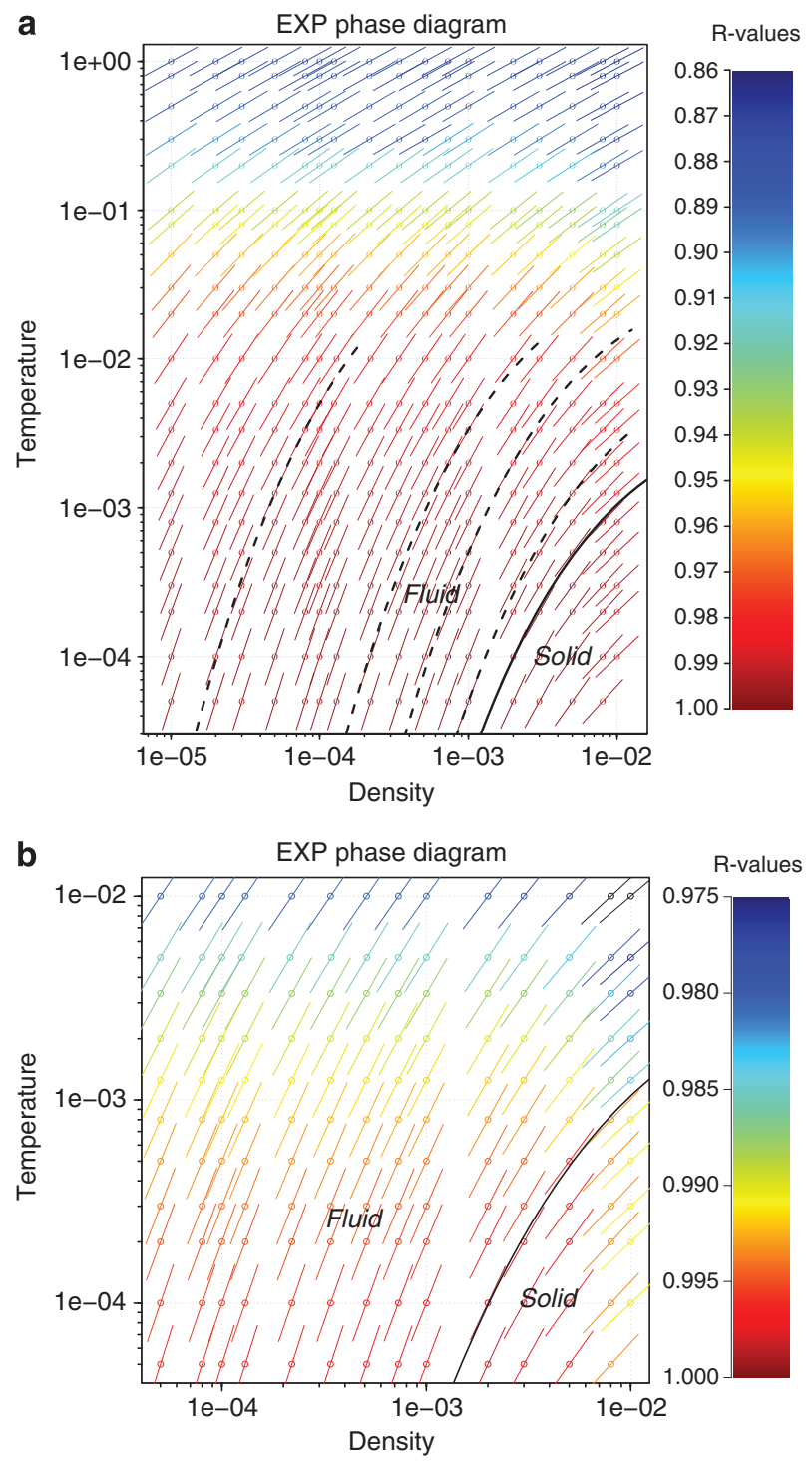

Figure 2 | Thermodynamic phase diagram of the EXP system. Density and temperature are both given in the unit system defined by pair-potential parameters (equation (1)). (a) gives an overview of the phase diagram and (b) zooms in on its low-temperature part; in both cases the colours indicate the value of the virial potential-energy correlation coefficient $R$ defined in equation (3). The slope of each line segment is that of the isomorph through the state point in question, the curve along which structure and dynamics are (approximately) invariant in reduced units. The full curves mark the melting isomorph; this curve's width in $\mathbf{a}$ is approximately that of the coexistence region. The dashed curves mark a few other isomorphs.

A pragmatic definition is that a given system is Roskilde simple at the state point in question if $R>0.9$ (ref. 58). This is the case for the EXP system whenever $k_{\mathrm{B}} T / \varepsilon<0.1$.

Figure $2 \mathrm{~b}$ zooms in on the low-temperature part of the phase diagram where the EXP system has particularly strong virial potential-energy correlations. In both figures the line segments have slope $\gamma$, the slope of the so-called isomorph through the state point in question that is calculated from the expression $\gamma=$ $\langle\Delta W \Delta U\rangle /\left\langle(\Delta U)^{2}\right\rangle$ (ref. 31). An isomorph is a curve in the phase diagram along which structure and dynamics to a good approximation are invariant in reduced units ${ }^{31,59}$. A system has isomorphs if and only if the system has strong virial potential- 


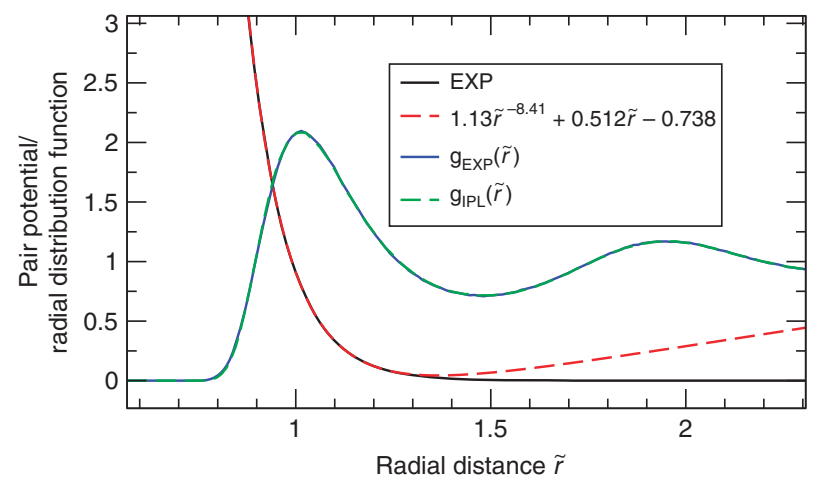

Figure 3 | Illustration of how the EXP pair potential may be fitted by an eIPL pair potential ${ }^{\mathbf{5 8}}$. The elPL pair potential (ref. 58) a sum of an inverse power-law (IPL) term and a linear term (red-dashed curve); $\tilde{r} \equiv r \rho^{1 / 3}$ is the reduced pair distance and the pair potential is given in units of $k_{\mathrm{B}} T$. The eIPL function was fitted to the EXP function over the range of radial distances for which the radial distribution function (RDF) at the state point defined by $\rho \sigma^{3}=1.0 \times 10^{-3}$ and $k_{\mathrm{B}} T / \varepsilon=5.0 \times 10^{-5}$ is larger than unity. The blue and green curves are the RDFs at this state point of the EXP pair potential and the IPL pair potential, respectively. The EXP and IPL pair potentials' diffusion constants differ by $<1 \%$ at this state point (data not shown).

energy correlations at the relevant state points ${ }^{31}$. The existence of isomorphs implies that the phase diagram becomes effectively one-dimensional for many physical properties; ref. 57 gives a recent review of the isomorph theory with a focus on its validation in computer simulations and experiments. The curves in Fig. 2a are examples of isomorphs of which the full curve is the melting isomorph. ${ }^{31}$ Isomorphs of the EXP system were studied briefly in ref. 40 .

Before proceeding we address the question why the EXP pair potential has strong virial potential-energy correlations. Our explanation refers to IPL pair potentials, which by Euler's theorem for homogeneous functions have $100 \%$ virial potential-energy correlations (recall that the microscopic virial is defined generally by $W(\mathbf{R})=(-1 / 3) \mathbf{R} \cdot \nabla U(\mathbf{R})$ (ref. 5)). Figure 3 refers to the state point $\rho \sigma^{3}=1.0 \times 10^{-3}, k_{\mathrm{B}} T / \varepsilon=5.0 \times 10^{-5}$. The figure shows that the EXP pair potential (black curve) may be fitted very well over the entire first coordination shell by an extended IPL ('eIPL') function, which is defined as an IPL term plus a linear term (the red-dashed line). As shown in ref. 58 the linear term contributes little to neither the virial nor the potential-energy constant-volume fluctuations because the sum of nearest-neighbour distances is almost constant. This is because if one particle is moved, some nearest-neighbour distances decrease and others increase, resulting in almost no change in the sum of the nearest-neighbour distances (this argument is exact in one dimension). As a consequence, the WU fluctuations are dominated by the IPL term and thus strongly correlating. This explains why the EXP pair potential has very strong WU correlations. To confirm the equivalence between the EXP pair potential and the IPL pair potential $\left(1.13 \tilde{r}^{-8.41}\right)$, we performed a simulation with the IPL pair potential at the same state point. As predicted $^{58,60}$ there is good agreement between the two systems' structure (blue and green curves) and dynamics (not shown), confirming that the linear term of the eIPL pair potential fitting the EXP pair potential, $0.512 \tilde{r}-0.738$, is not important for the physics.

Derivation of quasi-universality. We proceed to show that the strong virial potential-energy correlation property of the EXP system implies quasi-universality for a large class of monatomic systems. As shown recently ${ }^{39}$, any Roskilde-simple system, that is, with strong virial potential-energy correlations and isomorphs, is characterized by 'hidden-scale-invariance' in the sense that two functions of density exist, $h(\rho)$ and $g(\rho)$, such that the potentialenergy function $U(\mathbf{R})$ can be expressed as follows

$$
U(\mathbf{R}) \cong h(\rho) \tilde{\Phi}(\tilde{\mathbf{R}})+g(\rho) .
$$

Here $\tilde{\Phi}(\tilde{\mathbf{R}})$ is a dimensionless, state-point-independent function of the reduced dimensionless configuration vector $\tilde{\mathbf{R}} \equiv \rho^{1 / 3} \mathbf{R}$. In particular, equation (4) implies the strong correlations between the potential energies of scaled and unscaled configurations documented in Fig. 1: consider two configurations $\mathbf{R}_{1}$ and $\mathbf{R}_{2}$ at density $\rho_{1}$ and $\rho_{2}$, respectively, with the same reduced coordinates, that is, obeying $\rho_{1}^{1 / 3} \mathbf{R}_{1}=\rho_{2}^{1 / 3} \mathbf{R}_{2}$. By elimination of $\tilde{\Phi}(\tilde{\mathbf{R}})$ in equation (4) we get $U\left(\mathbf{R}_{2}\right) \cong\left(h\left(\rho_{2}\right) /\right.$ $\left.h\left(\rho_{1}\right)\right) U\left(\mathbf{R}_{1}\right)+g\left(\rho_{2}\right)-g\left(\rho_{1}\right) h\left(\rho_{2}\right) / h\left(\rho_{1}\right)$. Thus any two configurations with the same reduced coordinates have potential energies that are approximately linear functions of each other with constants that only depend on the two densities in question, see Fig. 1.

According to equation (4) a change of density implies that the potential-energy landscape to a good approximation undergoes a linear, affine transformation. Moreover, equation (4) implies invariant structure and dynamics in reduced units along the curves defined by constant $h(\rho) / k_{\mathrm{B}} T$, the equation identifying the isomorphs ${ }^{31,39,57}$ : along any curve defined by $h(\rho) / k_{\mathrm{B}} T=C$ the reduced force $\tilde{\mathbf{F}} \equiv-\tilde{\nabla} U(\mathbf{R}) / k_{B} T$ is given by $\tilde{\mathbf{F}}=-C \tilde{\nabla} \tilde{\Phi}(\tilde{\mathbf{R}})$, which implies that $\tilde{\mathbf{F}}$ is a certain function of the reduced coordinates, that is, $\tilde{\mathbf{F}}=\tilde{\mathbf{F}}(\tilde{\mathbf{R}})$. Since Newton's second law in reduced coordinates is $d^{2} \tilde{\mathbf{R}} / d \tilde{t}^{2}=\tilde{\mathbf{F}}$ (the reduced mass is unity), it follows that the particles move in the same way at different isomorphic state points-except for the trivial linear uniform scalings of space and time involved in transforming back to real units.

Since the EXP pair potential has strong virial potential-energy correlations, equation (4) implies that functions $h_{\mathrm{EXP}}(\rho), g_{\mathrm{EXP}}(\rho)$ and $\tilde{\Phi}_{\mathrm{EXP}}(\tilde{\mathbf{R}})$ exist such that

$$
U_{\mathrm{EXP}}(\mathbf{R}) \cong h_{\mathrm{EXP}}(\rho) \tilde{\Phi}_{\mathrm{EXP}}(\tilde{\mathbf{R}})+g_{\mathrm{EXP}}(\rho) .
$$

The functions $h_{\mathrm{EXP}}(\rho)$ and $g_{\mathrm{EXP}}(\rho)$ in equation (5) both have dimension energy. They can therefore be written as $h_{\mathrm{EXP}}(\rho)=$ $\varepsilon \tilde{h}_{\mathrm{EXP}}\left(\rho \sigma^{3}\right)$ and $g_{\mathrm{EXP}}(\rho)=\varepsilon \tilde{g}_{\mathrm{EXP}}\left(\rho \sigma^{3}\right)$ in which $\tilde{h}_{\mathrm{EXP}}$ and $\tilde{g}_{\mathrm{EXP}}$ are dimensionless functions that only depend on the dimensionless density $\rho \sigma^{3}$.

Consider now a pair potential $v(r)$ that can be expressed as follows

$$
v(r)=\int_{0}^{\infty} f(\sigma) e^{-r / \sigma} d \sigma .
$$

The system's potential energy is the sum of the individual pairpotential contributions with the same weights as in equation (6). Therefore, if $U(\mathbf{R})$ is the potential energy and we define $h(\rho) \equiv$ $\int_{0}^{\infty} f(\sigma) \tilde{h}_{\mathrm{EXP}}\left(\rho \sigma^{3}\right) d \sigma$ and $g(\rho) \equiv \int_{0}^{\infty} f(\sigma) \tilde{g}_{\mathrm{EXP}}\left(\rho \sigma^{3}\right) d \sigma$, it follows from equation (5) that

$$
U(\mathbf{R}) \cong h(\rho) \tilde{\Phi}_{\mathrm{EXP}}(\tilde{\mathbf{R}})+g(\rho) .
$$

Because the reduced-unit physics is encoded in the function $\tilde{\Phi}_{\mathrm{EXP}}(\tilde{\mathbf{R}})$ via Newton's equation $d^{2} \tilde{\mathbf{R}} / d \tilde{t}^{2}=-C \tilde{\nabla} \tilde{\Phi}_{\mathrm{EXP}}(\tilde{\mathbf{R}})$ where $C=h(\rho) / k_{\mathrm{B}} T$ identifes the isomorph through the state point in question, equation (7) implies identical structure and dynamics to a good approximation for systems obeying equation (6). This argument would be exact if the EXP pair potential had $100 \%$ virial potential-energy correlations. This is not the case, though, and the approximation equation (7) is only useful when it primarily involves EXP functions from the low- 
temperature part of the phase diagram where strong virial potential-energy correlations and thus equation (4) apply.

We have shown that a pair potential $v(r)$ is quasi-universal if it can be written as a sum of low-temperature EXP pair potentials. To translate this into an operational criterion, note the following. If the integral in equation (6) is discretized into a finite sum and expressed in terms of the reduced pair potential $\tilde{v} \equiv v / k_{B} T$ regarded as a function of the reduced pair distance $\left(\tilde{r} \equiv \rho^{1 / 3} r\right)$, the condition for quasi-universality is that

$$
\tilde{v}(\tilde{r}) \cong \sum_{j} \Lambda_{j} e^{-u_{j} \tilde{r}},\left|\Lambda_{j}\right| \gg 1 .
$$

It is understood that the 'wavevectors' $u_{j}$ are not so closely spaced that large positive and negative neighbouring terms may almost cancel one another. Several points should be noted: (1) Equation (8) is state-point dependent because the function $\tilde{v}(\tilde{r})$ varies with state point; (2) a continuous integral of EXP functions does not automatically obey equation (8)-it is necessary that the integral can be approximated by a finite sum of EXP terms, each with a numerically large prefactor; (3) a sum or product of two pair potentials obeying equation (8) with all $\Lambda_{j}>0$ gives a function that also obeys this equation.

Important examples. Consider first the IPL pair potential $v_{n}(r) \equiv \varepsilon(r / \sigma)^{-n}$. In terms of the reduced radius $\tilde{r}$, the reduced IPL pair potential is given by $\tilde{v}_{n}(\tilde{r}) \equiv v_{n}(r) / k_{B} T=\Gamma_{n} \tilde{r}^{-n}$ in which $\Gamma_{n} \equiv\left(\rho \sigma^{3}\right)^{n / 3} \varepsilon / k_{\mathrm{B}} T$. The mathematical identity $\int_{0}^{\infty} x^{n-1} \exp (-x) d x=(n-1)$ ! implies that $\tilde{v}_{n}(\tilde{r})=\left[\Gamma_{n} /\right.$ $(n-1) !] \int_{0}^{\infty} u^{n-1} \exp (-u \tilde{r}) d u$. Discretizing the integral leads to $\tilde{v}_{n}(\tilde{r}) \cong\left[\Gamma_{n} /(n-1) !\right] \Delta u \sum_{j=0}^{\infty}((j+1 / 2) \Delta u)^{n-1}$ $\exp (-(j+1 / 2) \Delta u \tilde{r})$. Writing $((j+1 / 2) \Delta u)^{n-1}=\exp [(n-1)$ $\ln ((j+1 / 2) \Delta u)]$, by differentiation with respect to $j$ it is easy to see that the dominant contributions to the sum come from the terms with $(n-1) /(j+1 / 2) \simeq \Delta u \tilde{r}$. Thus for typical nearestneighbour distances $(\tilde{r} \simeq 1)$ the terms with $(j+1 / 2) \Delta u \simeq n-1$ are the most important ones. For these values of $j$ the prefactor of the exponential in the above sum is roughly $\Gamma_{n} \Delta u(n-1)^{n-1 /}$ $(n-1)$ !. The largest realistic discretization step $\Delta u$ is in order of unity, so we conclude that for values of $n$ larger than three or four equation (8) is obeyed unless $\Gamma_{n}$ is very small, a condition that applies for the state points that have typically been studied ${ }^{9,20-23}$. The case of a Coulomb repulsive system $(n=1)$ is discussed in the next section.

As a consequence of the above, at most state points the potential energy of the IPL system, $U_{n}(\mathbf{R})$, can be written as

$$
U_{n}(\mathbf{R}) \cong h_{n}(\rho) \tilde{\Phi}_{\mathrm{EXP}}(\tilde{\mathbf{R}})+g_{n}(\rho) \text {. }
$$

Since $U_{n}(\mathbf{R}) \propto \rho^{n / 3}$ for the density variation induced by a uniform scaling of a configuration $\mathbf{R}$, that is, keeping $\tilde{\mathbf{R}}$ constant, one has $h_{n}(\rho) \propto \rho^{n / 3}$ and $g_{n}(\rho) \propto \rho^{n / 3}$. This means that two numbers $\alpha_{n}$ and $\beta_{n}$ exist such that $U_{n}(\mathbf{R}) \cong \varepsilon\left[\alpha_{n}\left(\rho \sigma^{3}\right)^{n / 3} \tilde{\Phi}_{\mathrm{EXP}}(\tilde{\mathbf{R}})+\beta_{n}\left(\rho \sigma^{3}\right)^{n / 3}\right]$. For a general pair potential of the form $v(r)=\varepsilon \sum_{n} v_{n}(r / \sigma)^{-n}$, by a linear combination of equation (9) we arrive at equation (7) in which $h(\rho)=$ $\varepsilon \sum_{n} v_{n} \alpha_{n}\left(\rho \sigma^{3}\right)^{n / 3}$ and $g(\rho)=\varepsilon \sum_{n} v_{n} \beta_{n}\left(\rho \sigma^{3}\right)^{n / 3}$.

A well-known case is the LJ pair potential $v_{\mathrm{LJ}}(r)=$ $4 \varepsilon\left[(r / \sigma)^{-12}-(r / \sigma)^{-6}\right]$. Consider the LJ liquid state point given by $\rho \sigma^{3}=1, k_{\mathrm{B}} T=2 \varepsilon$. In terms of the above-defined reduced IPL functions, it is easy to see that this is of the form equation (8), implying quasi-universality of the LJ liquid at this state point.

An application of the EXP theory of quasi-universality is the intriguing 'additivity of melting temperatures' ${ }^{61}$ according to which if two systems have melting temperatures that as functions of density are denoted by $T_{\mathrm{m}, 1}(\rho)$ and $T_{\mathrm{m}, 2}(\rho)$, the melting temperature of the system with the sum potential energy is
$T_{\mathrm{m}, 1}(\rho)+T_{\mathrm{m}, 2}(\rho)$. This property follows from quasi-universality because the dynamics of all three systems are controlled by the same function $\tilde{\Phi}_{\mathrm{EXP}}(\tilde{\mathbf{R}})$. For the EXP system melting initiates when this function's average upon heating reaches a certain value, and the same must apply for all quasi-universal systems. In particular, since for an IPL system one has $T_{\mathrm{m}}(\rho) \propto \rho^{n / 3}$ (ref. 62), the melting temperature of the LJ system varies with density according to the expression $T_{\mathrm{m}}(\rho)=A \rho^{4}-B \rho^{2}$ (refs 61,63).

\section{Discussion}

Denominating the systems that obey equation (8) collectively as the EXP quasi-universality class, we have shown that all systems in this class are quasi-universal in the sense of this paper: if a single reduced-unit structural or dynamic quantity is known, all other reduced-unit structural or dynamic quantities are known to a good approximation. This is because these are all encoded in the function $\Phi_{\mathrm{EXP}}(\tilde{\mathbf{R}})$, and any reduced-unit quantity characterizing structure or dynamics identifies the constant $C=h(\rho) / k_{\mathrm{B}} T$ of the reduced-unit version of Newton's second law $d^{2} \tilde{\mathbf{R}} / d \tilde{t}^{2}=-C \tilde{\nabla} \tilde{\Phi}_{\mathrm{EXP}}(\tilde{\mathbf{R}})$.

The obvious question is whether all quasi-universal systems are in the EXP quasi-universality class. We cannot prove this, but conjecture it is the case based on the available evidence in the literature for the following systems:

The Jagla pair potential is a HS potential plus a finite-width potential well defined by two terms that are linear in $r$ (ref. 37). This pair potential is not in the EXP quasi-universality class-it cannot be approximated as a sum of exponentials because its Laplace transform only has a pole at zero. Indeed, the Jagla pair potential reproduces water's anomalous density maximum and has a liquid-liquid critical point ${ }^{19}$, properties which are both inconsistent with quasi-universality.

The Gaussian core model (GCM) is a Gaussian centred at $r=0$. For this system there is a re-entrant body-centered cubic phase above the triple point ${ }^{15}$ and the transport coefficients have a non-monotonous density dependence at constant temperature $^{18}$. These observations both contradict quasiuniversality. Consistent with the above conjecture, a representation of the form $\exp \left(-r^{2} / 2 \sigma^{2}\right)=\int_{0}^{\infty} \phi(u) e^{-u r} d u$ does not exist because the Laplace transform of a Gaussian has no poles; thus the GCM system is not in the EXP quasiuniversality class. At low temperatures and low densities, the GCM pair potential can be approximated well by an exponential, however, and in this part of the phase diagram the GCM system is indeed quasi-universal ${ }^{11,15}$.

The LJ Gaussian (LJG) model is a pair potential that is arrived at by adding a negative, displaced Gaussian to the LJ pair potential $^{17}$. Like the GCM it cannot be written as a sum of exponentials. The LJG system has thermodynamic and dynamic anomalies ${ }^{16}$ and 'a surprising variety of crystals' ${ }^{17}$, both of which are observations that violate quasi-universality.

The OCP is the common name for the single-charge Coulomb system. The Coulomb potential is too long ranged for a thermodynamic limit to exist for the free energy per particle unless a uniform charge-compensating background is introduced $^{5,49}$. Nevertheless, any finite OCP system is well-defined and amenable to computer simulation-in fact the OCP system was an important example in Rosenfeld's original paper on excess-entropy scaling ${ }^{6}$. The OCP system is the $n=1$ case of the above discussed IPL pair potential; it is quasi-universal in the dense fluid case, that is, whenever $\Gamma_{1} \gg 1$ (writing $\tilde{v}_{1} \equiv \Gamma_{1} / \tilde{r}$ ). Violations of quasi-universality are indeed known to gradually appear when $\Gamma_{1}$ goes below 50 (ref. 64).

The Yukawa pair potential is given by the expression $v(r)=$ $\varepsilon(\sigma / r) \exp (-r / \sigma)$. Since $v(r) / \varepsilon=\int_{0}^{\infty} \exp (-r(u+1) / \sigma) d u$ it is 
easy to see that this pair potential is in the EXP quasi-universality class whenever $k_{\mathrm{B}} T \ll \varepsilon$. This is consistent with known properties $^{65}$.

This paper has argued that one may replace the HS by the EXP system as the generic simple pair-potential system from which quasi-universality is derived. We do not suggest that the HS system's role in liquid-state physics is entirely undeserved, however, because this model is still uniquely simple and mathematically beautiful. Nevertheless, by using the EXP pair potential as the fundamental building block, a number of advantages are obtained. First of all, smoothness is ensured. Second, if the conjecture that all quasi-universal functions are in the EXP quasi-universality class is confirmed, we now have a mathematically precise characterization of all quasi-universal pair potentials (equation (8)). Third, the present explanation of quasiuniversality gives a natural explanation of dynamic quasiuniversality. Finally, the role of the HS pair potential in liquidstate physics is clarified: quasi-universality is not caused by a given system's similarity to the HS system-rather, quasiuniversality implies similarity between the properties of many systems and those of the HS system simply because the latter is in the EXP quasi-universality class by being the $n \rightarrow \infty$ limit of an IPL system. Note that while this paper focused on quasiuniversality for liquid models, the crystalline phases of these models are likewise quasi-universal, for instance by having quasiuniversal radial distribution functions, phonon spectra and vacancy jump dynamics ${ }^{66}$.

In continuing work it will be interesting to investigate the consequences of the EXP approach for the fact that quasiuniversality appears to apply beyond the framework of thermal equilibrium, for example, for the jamming transition in which the HS model is presently used as the generic model ${ }^{67}$. It is an open question whether replacing the HS model by the EXP model in thermodynamic perturbation theory would have advantages ${ }^{5}$. Another open question is to which extent mixtures are quasiuniversal $^{68}$. Finally, recent results from the works of Truskett and co-workers ${ }^{11}$ show that classical Rosenfeld excess-entropy scaling may be modified into more general excess-entropy scalings, and it would be interesting to investigate whether the present approach can somehow be extended to account for this.

\section{Method}

Simulation system. A system of $N=1,000$ particles interacting via the EXP pair potential was simulated using standard Nose-Hoover NVT simulations with a time step of 0.0025 and thermostat relaxation time of 0.2 (LJ units). A shiftedforces cut-off at $r=2 \rho^{-1 / 3}$ was used for densities below $1.0 \times 10^{-3}$, at larger densities the cut-off was $4 \rho^{-1 / 3}$. At each state point the simulations involved $10,000,000$ time steps after equilibration. For temperatures below $2.0 \times 10^{-3}$, the simulations were initiated from a state of 2,000 particles placed in a body-centred cubic crystal structure. The melting isomorph was determined by the interface pinning method ${ }^{69}$ - the NPT simulations involved here were made using LAMMPS $^{70}$ (http://lammps.sandia.gov) with shifted-potential cut-offs ranging from $2.5 \rho^{-1 / 3}$ to $4.0 \rho^{-1 / 3}$ for a system of 2,560 particles; the time step was 0.005 and the relaxation time was 0.4 for the thermostat and 0.8 for the barostat.

\section{References}

1. Bernal, J. D. A geometrical approach to the structure of liquids. Nature 183, 141-147 (1959).

2. Alder, B. J. \& Wainwright, T. E. Phase transition for a hard sphere system. J. Chem. Phys. 27, 1208-1209 (1957).

3. Weeks, J. D., Chandler, D. \& Andersen, H. C. Role of repulsive forces in determining the equilibrium structure of simple liquids. J. Chem. Phys. 54, 5237-5247 (1971)

4. Barker, J. A. \& Henderson, D. What is "liquid"? Understanding the states of matter. Rev. Mod. Phys. 48, 587-671 (1976).

5. Hansen, J.-P. \& McDonald, I. R. Theory of Simple Liquids: With Applications to Soft Matter, 4th edn (Academic, 2013).

6. Rosenfeld, Y. Relation between the transport coefficients and the internal entropy of simple systems. Phys. Rev. A 15, 2545-2549 (1977).
7. Krekelberg, W. P., Mittal, J., Ganesan, V. \& Truskett, T. M. How short-range attractions impact the structural order, self-diffusivity, and viscosity of a fluid. J. Chem. Phys. 127, 044502 (2007).

8. Chakraborty, S. N. \& Chakravarty, C. Entropy, local order, and the freezing transition in Morse liquids. Phys. Rev. E 76, 011201 (2007).

9. Branka, A. C. \& Heyes, D. M. Pair correlation function of soft-sphere fluids. J. Chem. Phys. 134, 064115 (2011).

10. de J. Guevara-Rodríguez, F. \& Medina-Noyola, M. Dynamic equivalence between soft- and hard-core Brownian fluids. Phys. Rev. E 68, 011405 (2003).

11. Pond, M. J., Errington, J. R. \& Truskett, T. M. Generalizing Rosenfeld's excess-entropy scaling to predict long-time diffusivity in dense fluids of brownian particles: from hard to ultrasoft interactions. J. Chem. Phys. 134, 081101 (2011).

12. Schmiedeberg, M., Haxton, T. K., Nagel, S. R. \& Liu, A. J. Mapping the glassy dynamics of soft spheres onto hard-sphere behavior. Europhys. Lett. 96, 36010 (2011).

13. Lopez-Flores, L. et al. Dynamic equivalence between atomic and colloidal liquids. Europhys. Lett. 99, 46001 (2012).

14. Lopez-Flores, L., Ruiz-Estrada, H., Chavez-Paez, M. \& Medina-Noyola, M. Dynamic equivalences in the hard-sphere dynamic universality class. Phys. Rev. E 88, 042301 (2013).

15. Prestipino, S., Saija, F. \& Giaquinta, P. V. Phase diagram of softly repulsive systems: the Gaussian and inverse-power-law potentials. J. Chem. Phys. 123, 144110 (2005).

16. Barros de Oliveira, A., Netz, P. A., Colla, T. \& Barbosa, M. C. Thermodynamic and dynamic anomalies for a three-dimensional isotropic core-softened potential. J. Chem. Phys. 124, 084505 (2006).

17. Engel, M. \& Trebin, H.-R. Self-assembly of monatomic complex crystals and quasicrystals with a double-well interaction potential. Phys. Rev. Lett. 98, 225505 (2007).

18. Krekelberg, W. P. et al. Generalized Rosenfeld scalings for tracer diffusivities in not-so-simple fluids: mixtures and soft particles. Phys. Rev. E 80, 061205 (2009).

19. Gallo, P. \& Sciortino, F. Ising universality class for the liquid-liquid critical point of a one component fluid: A finite-size scaling test. Phys. Rev. Lett. 109, 177801 (2012).

20. Heyes, D. M. \& Branka, A. C. The influence of potential softness on the transport coefficients of simple fluids. J. Chem. Phys. 122, 234504 (2005).

21. Scopigno, T. et al. Hard-sphere-like dynamics in a non-hard-sphere liquid. Phys. Rev. Lett. 94, 155301 (2005).

22. Branka, A. C. \& Heyes, D. M. Thermodynamic properties of inverse power fluids. Phys. Rev. E 74, 031202 (2006).

23. Heyes, D. M. \& Branka, A. C. Self-diffusion coefficients and shear viscosity of inverse power fluids: from hard- to soft-spheres. Phys. Chem. Chem. Phys. 10, 4036-4044 (2008).

24. Young, T. \& Andersen, H. C. A scaling principle for the dynamics of density fluctuations in atomic liquids. J. Chem. Phys. 118, 3447-3450 (2003).

25. Truskett, T. M., Torquato, S. \& Debenedetti, P. G. Towards a quantification of disorder in materials: distinguishing equilibrium and glassy sphere packings. Phys. Rev. E 62, 993-1001 (2000).

26. Ubbelohde, A. R. Melting and Crystal Structure (Clarendon, 1965).

27. Khrapak, S. A., Chaudhuri, M. \& Morfill, G. E. Communication: universality of the melting curves for a wide range of interaction potentials. J. Chem. Phys. 134, 241101 (2011).

28. Andrade, E. N. C. A theory of the viscosity of liquids-Part I. Phil. Mag. 17, 497-511 (1934).

29. Hansen, J.-P. \& Verlet, L. Phase transitions of the Lennard-Jones system. Phys. Rev. 184, 151-161 (1969).

30. Baus, M. The modern theory of crystallization and the Hansen-Verlet rule. Mol. Phys. 50, 543-565 (1983)

31. Gnan, N., Schrøder, T. B., Pedersen, U. R., Bailey, N. P. \& Dyre, J. C. Pressureenergy correlations in liquids. IV. "isomorphs" in liquid phase diagrams. J. Chem. Phys. 131, 234504 (2009).

32. Pond, M. J., Errington, J. R. \& Truskett, T. M. Mapping between long-time molecular and Brownian dynamics. Soft Matter 7, 9859-9862 (2011).

33. Young, T. \& Andersen, H. C. Tests of an approximate scaling principle for dynamics of classical fluids. J. Phys. Chem. B 109, 2985-2994 (2005).

34. Rosenfeld, Y. A quasi-universal scaling law for atomic transport in simple fluids. J. Phys. Condens. Matter 11, 5415-5427 (1999).

35. Stillinger, F. H. Phase transitions in the Gaussian core system. J. Chem. Phys. 65, 3968-3974 (1976).

36. Zachary, C. E., Stillinger, F. H. \& Torquato, S. Gaussian core model phase diagram and pair correlations in high euclidean dimensions. J. Chem. Phys. 128, 224505 (2008)

37. Yan, Z., Buldyrev, S. V., Giovambattista, N., Debenedetti, P. G. \& Stanley, H. E. Family of tunable spherically symmetric potentials that span the range from hard spheres to waterlike behavior. Phys. Rev. E 73, 051204 (2006). 
38. Dyre, J. C. NVU perspective on simple liquids' quasiuniversality. Phys. Rev. E 87, 022106 (2013).

39. Dyre, J. C. Isomorphs, hidden scale invariance, and quasiuniversality. Phys. Rev. E 88, 042139 (2013)

40. Bacher, A. K. \& Dyre, J. C. The mother of all pair potentials. Colloid Polym. Sci. 292, 1971-1975 (2014).

41. Born, M. \& Meyer, J. E. Zur Gittertheorie der Ionenkristalle. Z. Phys. 75, 1-18 (1932).

42. Buckingham, R. A. The classical equation of state of gaseous helium, neon and argon. Proc. R. Soc. Lond. A 168, 264-283 (1938).

43. Girifalco, L. A. \& Weizer, V. G. Application of the Morse potential function to cubic metals. Phys. Rev. 114, 687-690 (1959).

44. Buckingham, R. A. \& Corner, J. Tables of second virial and low-pressure JouleThompson coefficients for intermolecular potentials with exponential repulsion. Proc. R. Soc. A 189, 118-129 (1947).

45. Mason, E. A. \& Rice, W. E. The intermolecular potentials for some simple nonpolar molecules. J. Chem. Phys. 22, 843-851 (1954).

46. Abrahamson, A. A. Born-Mayer-type interatomic potential for neutral groundstate atoms with $Z=2$ to $Z=105$. Phys. Rev. 178, 76-79 (1969).

47. Gupta, N. P. Interpretation of phonon dispersion in solid argon and neon. J. Solid State Chem. 5, 477-480 (1972).

48. Toda, A. Nonlinear Waves and Solitons (Kluwer, 1983).

49. Hansen, J.-P., McDonald, I. R. \& Pollock, E. L. Statistical mechanics of dense ionized matter. III. Dynamical properties of the classical one-component plasma. Phys. Rev. A 11, 1025-1039 (1975).

50. Ingebrigtsen, T. S., Schrøder, T. B. \& Dyre, J. C. Isomorphs in model molecular liquids. J. Phys. Chem. B 116, 1018-1034 (2012).

51. Veldhorst, A. A., Dyre, J. C. \& Schrøder, T. B. Scaling of the dynamics of flexible Lennard-Jones chains. J. Chem. Phys. 141, 054904 (2014).

52. Malins, A., Eggers, J. \& Royall, C. P. Investigating isomorphs with the topological cluster classification. J. Chem. Phys. 139, 234505 (2013).

53. Prasad, S. \& Chakravarty, C. Onset of simple liquid behaviour in modified water models. J. Chem. Phys. 140, 164501 (2014).

54. Flenner, E., Staley, H. \& Szamel, G. Universal features of dynamic heterogeneity in supercooled liquids. Phys. Rev. Lett. 112, 097801 (2014).

55. Henao, A., Pothoczki, S., Canales, M., Guardia, E. \& Pardo, L. C. Competing structures within the first shell of liquid $\mathrm{C}_{2} \mathrm{Cl}_{6}$ : a molecular dynamics study. J. Mol. Liq. 190, 121-125 (2014)

56. Pieprzyk, S., Heyes, D. M. \& Branka, A. C. Thermodynamic properties and entropy scaling law for diffusivity in soft spheres. Phys. Rev. E 90, 012106 (2014).

57. Dyre, J. C. Hidden scale invariance in condensed matter. J. Phys. Chem. B 118, 10007-10024 (2014)

58. Bailey, N. P., Pedersen, U. R., Gnan, N., Schrøder, T. B. \& Dyre, J. C. Pressureenergy correlations in liquids. II. analysis and consequences. J. Chem. Phys. 129, 184508 (2008)

59. Ingebrigtsen, T. S., Schrøder, T. B. \& Dyre, J. C. What is a simple liquid? Phys. Rev. X 2, 011011 (2012).
60. Pedersen, U. R., Schrøder, T. B. \& Dyre, J. C. Repulsive reference potential reproducing the dynamics of a liquid with attractions. Phys. Rev. Lett. 105, 157801 (2010).

61. Rosenfeld, Y. Universality of melting and freezing indicators and additivity of melting curves. Mol. Phys. 32, 963-977 (1976).

62. Stishov, S. M. The thermodynamics of melting of simple substances. Sov. Phys Usp. 17, 625-643 (1975).

63. Khrapak, S. A. \& Morfill, G. E. Accurate freezing and melting equations for the Lennard-Jones system. J. Chem. Phys. 134, 094108 (2011).

64. Daligault, J. Liquid-state properties of a one-component plasma. Phys. Rev. Lett. 96, 065003 (2006).

65. Rosenfeld, Y. Quasi-universal melting-temperature scaling of transport coefficients in Yukawa systems. J. Phys. Condens. Matter 13, L39-L43 (2001).

66. Albrechtsen, D. E., Olsen, A. E., Pedersen, U. R., Schrøder, T. B. \& Dyre, J. C. Isomorph invariance of the struccture and dynamics of classical crystals. Phys. Rev. B 90, 094106 (2014).

67. Lerner, E., Düring, G. \& Wyart, M. Toward a microscopic description of flow near the jamming threshold. Europhys. Lett. 99, 58003 (2012).

68. Lerner, E., Bailey, N. P. \& Dyre, J. C. Density scaling and quasiuniversality of flow-event statistics for athermal plastic flows. Phys. Rev. E.: Preprint at http:// arxiv.org/pdf/1405.0156.pdf (2014).

69. Pedersen, U. R. Direct calculation of the solid-liquid Gibbs free energy difference in a single equilibrium simulation. J. Chem. Phys. 139, 104102 (2013).

70. Plimpton, S. Fast parallel algorithms for short-range molecular dynamics. J. Comp. Phys. 117, 1-19 (1995).

\section{Acknowledgements}

We are indebted to Ulf Pedersen for help in determining the freezing and melting isomorphs. The center for viscous liquid dynamics 'Glass and Time' is sponsored by the Danish National Research Foundation's grant DNRF61.

\section{Author contributions}

A.K.B. and T.B.S. performed the simulations and carried out the data analysis; J.C.D conceived the project and wrote the paper.

\section{Additional information}

Competing financial interests: The authors declare no competing financial interests

Reprints and permission information is available online at http://npg.nature.com/ reprintsandpermissions/

How to cite this article: Bacher, A. K. et al. Explaining why simple liquids are quasiuniversal. Nat. Commun. 5:5424 doi: 10.1038/ncomms6424 (2014). 